\title{
Minimalinvasive Chirurgie bei Malignomen des Gastrointestinaltrakts: Osophagus - Kontra-Position
}

\author{
Ralf Gertler Marcus Feith \\ Chirurgische Klinik und Poliklinik, Klinikum rechts der Isar, Technische Universität München, Deutschland
}

Schlüsselwörter

Ösophaguskarzinom · Minimalinvasive Ösophagektomie · Offene Ösophagektomie

\section{Zusammenfassung}

Hintergrund: Die chirurgische Therapie des Ösophaguskarzinoms besteht in der Ösophagektomie mit Lymphadenektomie. Diese wird traditionell offen, in den letzten Jahren jedoch auch zunehmend minimalinvasiv durchgeführt. Methoden: Ziel dieser Übersichtsarbeit ist es, die Kontra-Position zur minimalinvasiven Chirurgie darzulegen. Hierfür führten wir eine Literaturrecherche bei PubMed durch. Es wurden eine randomisiert kontrollierte Studie, vier Metaanalysen sowie 18 retrospektive Kohortenstudien einbezogen. Ergebnisse: Insgesamt zeigt sich bei den vorliegenden Publikationen ein niedriger Evidenzlevel mit anzunehmendem Selektionsbias in den retrospektiven Kohortenstudien. Trotzdem kann davon ausgegangen werden, dass die minimalinvasive Ösophagektomie heutzutage zumindest mit vergleichbarer Morbidität und Mortalität wie die offene Ösophagektomie durchgeführt werden kann und mit weniger Schmerzen, kürzerem Krankenhausaufenthalt und höherer früh-postoperativer Lebensqualität einhergeht. Unterschiedliche Ergebnisse zeigen sich bezüglich einer möglichen Reduktion von postoperativen pulmonalen Komplikationen nach der minimalinvasiven Operation, nicht zuletzt, weil hier neben dem reinen operativen Zugangsweg auch andere Faktoren wie die Ein-Seiten-Lungenbeatmung und eine längere Lungenkompression Einfluss nehmen. Schlussfolgerungen: Der onkologische Stellenwert der minimalinvasiven Ösophagektomie ist auf der Grundlage der vorliegenden Literatur nicht ausreichend zu beurteilen, auch wenn vergleichbare R0-Resektionsraten und Lymphknotenzahlen berichtet werden. Zu wenig standardisiert sind hier die durchgeführten Operationen, während die Patientenkollektive bezüglich Tumortyp, Stadienverteilung und Nachsorge zu heterogen ausfallen.

\section{Keywords}

Esophageal carcinoma - Minimally invasive esophagectomy . Open esophagectomy

\section{Summary}

Minimally Invasive Surgery for Malignancies of the Gastrointestinal Tract: Esophagus - Contra Position

Background: The standard therapy for esophageal carcinoma traditionally is the open esophagectomy with lymphadenectomy. In the last few years, however, minimally invasive approaches were increasingly used. Methods: This review describes the contra position regarding minimally invasive approaches for esophageal carcinoma. We performed a literature search for current studies in PubMed. A total of one randomized controlled trial, four systematic reviews, and 18 retrospective cohort analyses were included. Results: The evidence level of the retrieved studies is low, while selection bias must be suspected for the retrospective cohort analyses. However, it seems to be clear that minimally invasive esophagectomy can be performed with at least similar morbidity and mortality as the open resection. Moreover, the minimally invasive approach coincides with less pain, shorter hospital duration, and early postoperative quality of life. There is controversy whether minimally invasive surgery reduces pulmonary complications since other factors apart from the operative approach, such as single-lung ventilation and patient positioning, also influence respiratory outcome. Conclusions: The current literature does not allow for an adequate evaluation of the oncologic outcomes of minimally invasive esophagectomy, despite comparable R0 resection rates and lymph node harvest. In the available studies, only little standardization of the applied operations as well as a great heterogeneity of the patient cohorts with respect to tumor type, stage, and follow-up are to be found.

\section{KARGER \\ Fax +497614520714 \\ Information@Karger.com}

www.karger.com (c) 2013 S. Karger GmbH, Freiburg

1662-6664/13/0296-0350\$38.00/0

Accessible online at:

www.karger.com/vim
Prof. Dr. Marcus Feith

Chirurgische Klinik und Poliklinik

Klinikum rechts der Isar, Technische Universität München

Ismaninger Straße 22, 81675 München, Deutschland

marcus.feith@tum.de 


\section{Einleitung}

Die Ösophagektomie mit Lymphadenektomie stellt den Standard der chirurgischen Behandlung von Ösophaguskarzinomen dar. Je nach Tumortyp, funktionellem Risikoprofil und Tumorstadium wird die Operation als alleinige Therapie oder im Rahmen von multimodalen Therapiekonzepten eingesetzt. Trotz wesentlicher Fortschritte im perioperativen Management gehen Ösophagusresektionen nach wie vor mit einer erheblichen Morbidität und auch Mortalität einher. Angesichts des ausgedehnten Komplikationsspektrums von Ösophaguseingriffen und vor dem Hintergrund vielversprechender Ergebnisse beim Einsatz minimalinvasiver Operationsverfahren bei anderen malignen Erkrankungen ist es nicht verwunderlich, dass in den letzten Jahren auch für Malignome des Ösophagus minimalinvasive Operationstechniken entwickelt und zunehmend eingesetzt wurden.

Ziel dieser Übersichtsarbeit ist es, die aktuelle Studienlage zur minimalinvasiven Chirurgie im Vergleich mit der offenen Chirurgie bei Malignomen des Ösophagus methodisch und inhaltlich zu überprüfen. Wir beschreiben diesbezüglich die Kontra-Position zur minimalinvasiven Chirurgie. Entscheidende Parameter im Vergleich der Operationstechniken sind insbesondere die postoperative Morbidität und Mortalität, die chirurgische Radikaliät, das onkologische Outcome, die postoperative Lebensqualität sowie die Kosten.

\section{Vorgehensweise}

Wir führten eine Literaturrecherche mittels Medline und PubMed durch. Nur englischsprachige Publikationen, die einen Vergleich von minimalinvasiver Ösophagektomie mit offener Ösophagektomie beinhalten, wurden berücksichtigt. Fallserien, die nur über eine dieser Operationstechniken berichten, wurden ausgeschlossen. Der Evidenzlevel der Publikationen wurde gemäß einer modifizierten Klassifikation nach Sackett [1] beurteilt. Danach sind randomisierte kontrollierte Studien (randomized controlled trials (RCTs)) mit Fallzahlkalkulation und definiertem Studienendpunkt als Level I, RCTs ohne Fallzahlkalkulation oder ohne definierten Studienendpunkt als Level II, nicht randomisierte prospektive Studien als Level III und retrospektive Analysen und Kohortenstudien als Level IV zu klassifizieren. Da zum Thema nur eine Studie mit Evidenzlevel I [2] vorliegt, können die wichtigsten Ergebnisse der Publikationen im Folgenden lediglich in deskriptiver Weise dargestellt werden. Zudem wird auch auf die Ergebnisse der bislang zu dieser Fragestellung vorliegenden Metaanalysen eingegangen.

\section{Ergebnisse}

Insgesamt wurden eine RCT [2], vier systematische Reviews mit Metaanalysen [3-6] sowie 18 retrospektive Kohor- tenstudien [7-24] gefunden. Entsprechend liegt somit nur eine einzige Publikation mit Evidenzlevel I vor [2].

\section{Operationsverfahren}

Über alle Publikationen hinweg besteht eine ausgesprochene Heterogenität der angewandten Operationstechniken sowohl in der offenen als auch in der minimalinvasiven Technik. Zudem ist insbesondere das angewandte offene Operationsverfahren selbst innerhalb der einzelnen Publikationen nicht einheitlich verwendet worden und oft nur unpräzise definiert $[9,13,23]$. Dies trifft auch auf die einzige RCT zu, bei der unter anderem sowohl intrathorakale als auch zervikale Anastomosen ohne klare Definition der Indikation hierfür verwendet wurden [2]. Bei den minimalinvasiven Operationstechniken wurden sowohl total minimalinvasive Verfahren (laparoskopisch und thorakoskopisch) als auch Hybrid-Verfahren (Laparoskopie mit Thorakotomie oder Laparotomie mit Thorakoskopie) und laparoskopisch oder mediastinoskopisch assistierte transhiatale Verfahren eingesetzt.

\section{Intraoperative Beatmungstechnik und Patientenlagerung}

Heterogenität besteht zudem hinsichtlich der intraoperativen Beatmungstechnik, sowohl über alle Publikationen hinweg als auch innerhalb der einzelnen Publikationen. So wurde bei den offenen Operationen, soweit dezidiert beschrieben, ein Doppel-Lumen-Tubus mit intraoperativer linksseitiger EinLungen-Beatmung und Totalkollaps sowie manueller oder instrumenteller Kompression der rechten Lunge verwendet [2,9]. Bei den minimalinvasiven Operationen ist darüber hinaus auch die Verwendung von Single-Lumen-Tuben mit intraoperativ erhaltener Zwei-Lungen-Beatmung beschrieben [2]. Einzelne Autoren verwenden zudem eine Kohlendioxid-Insufflation bei der Thorakoskopie für einen Partialkollaps der rechten Lunge bei erhaltener rechtsseitiger Lungenventilation [2].

Auch bezüglich der Patientenlagerung für den thorakalen Part der Operation ist sowohl über alle Publikationen hinweg als auch innerhalb der einzelnen Publikationen eine Heterogenität zu verzeichnen. Während die verwendeten Thorakotomien in Linksseitenlagerung erfolgten, wurden die Thorakoskopien sowohl in Linksseitenlagerung als auch in Bauchlagerung durchgeführt.

\section{Operationsdauer, Blutverlust und}

\section{Krankenhausverweildauer}

Die meisten Publikationen (inklusive der RCT) zeigten eine längere Operationszeit für die minimalinvasive Chirurgie im Vergleich zur offenen Chirurgie (329 vs. 299 min) [2, 8, 1012, 15, 18, 21]; zwei Autoren berichteten gegenteilige Ergebnisse [7, 24]. Eine von vier Metaanalysen analysierte die Operationsdauer und fand keinen Unterschied zwischen minimalinvasiver und offener Chirurgie [5]. Nahezu bei allen Publikationen (inklusive der RCT) ging die minimalinvasive Technik mit weniger Blutverlust einher (200 vs. $475 \mathrm{ml}$ ) [2, 7, 9, 10, 12, $15,17,18,21,24]$. Eine von vier Metaanalysen analysierte den 
Blutverlust und zeigte einen geringeren Blutverlust bei minimalinvasiver Chirurgie [5]. Eine kürzere Krankenhausverweildauer zugunsten der minimalinvasiven Chirurgie zeigte sich sowohl in der RCT (11 vs. 14 Tage) [2] als auch bei fünf weiteren Publikationen $[9,14,17,18,21]$. Nur Parameswaran et al. [11] berichteten über gegenteilige Ergebnisse. Eine von vier Metaanalysen analysierte die Krankenhausverweildauer und fand eine kürzere Liegezeit bei minimalinvasiver Chirurgie [5].

\section{Postoperative Morbidität und Mortalität}

Als primäre Endpunkte der RCT definiert, zeigten sich bei der Studie von Biere et al. [2] nach minimalinvasiver Chirurgie im Vergleich zur offenen Chirurgie weniger pulmonale Infekte sowohl innerhalb der ersten beiden postoperativen Wochen (9 vs. 29\%) als auch während des gesamten Krankenhausaufenthalts (12 vs. 34\%). Vier Publikationen berichteten ebenfalls über weniger pulmonale Komplikationen nach minimalinvasiver Operation [10, 11, 14, 19]. Die Mehrzahl der verfügbaren Publikationen fand keine Unterschiede zwischen minimalinvasiver und offener Chirurgie bezüglich postoperativer pulmonaler Komplikationen [7-9, 12, 15, 17, 18, 20-24]. Drei von vier Metaanalysen analysierten pulmonale Komplikationen, wobei zwei Metaanalysen keinen Unterschied zwischen minimalinvasiver und offener Chirurgie fanden [3, 4], während eine Metaanalyse weniger respiratorische Komplikationen nach minimalinvasiver Operation beobachtete [5]. Nur Schoppmann et al. [14] berichteten über mehr Anastomosenprobleme nach offener Chirurgie im Vergleich zur minimalinvasiven Chirurgie (26 vs. 3\%); in allen übrigen Publikationen inklusive der RCT (7 vs. 12\%) ergaben sich keine signifikanten Unterschiede hinsichtlich der Anastomose. Drei von vier Metaanalysen analysierten Anastomoseninsuffizienzen, wobei zwei Metaanalysen keine Unterschiede zwischen minimalinvasiver und offener Chirurgie fanden [4, 5], während eine Metaanalyse weniger Anastomoseninsuffizienzen nach minimalinvasiver Chirurgie beobachtete [3]. Drei Publikationen zeigten mehr Rekurrensparesen nach minimalinvasiver Operation [11, 14, 20], während Biere et al. [2] in ihrer RCT mehr Rekurrensparesen nach der offenen Operation beobachteten (14 vs. $2 \%)$. Zwei von vier Metaanalysen analysierten Rekurrensparesen und fanden keinen Unterschied zwischen der minimalinvasiven und der offenen Chirurgie [4, 5]. Zwei Publikationen berichteten über eine höhere Krankenhausmortalität nach offener Operation gegenüber minimalinvasiver Operation [13, 20]; alle anderen Publikationen inklusive der RCT (2 vs. 3\%) zeigten keine Mortalitätsunterschiede. Alle vier Metaanalysen analysierten die 30-Tage-Mortaliät und fanden keinen Unterschied zwischen minimalinvasiver und offener Chirurgie [3-6].

\section{Chirurgisch-onkologische Radikalität}

Die Anzahl der entfernten Lymphknoten unterschied sich zwischen minimalinvasiver und offener Chirurgie bei vier Publikationen und der RCT nicht (20 vs. 21) [2, 8, 9, 14, 24], während in vier Publikationen durch die minimalinvasive
Vorgehensweise mehr Lymphknoten als bei den offenen Operationen entfernt wurden [11, 16, 17, 21]. Drei von vier Metaanalysen analysierten die Anzahl der entfernten Lymphknoten, wobei zwei Metaanalysen keinen Unterschied zwischen minimalinvasiver und offener Chirurgie fanden $[4,5]$, während eine Metaanalyse eine höhere Anzahl an entfernten Lymphknoten bei der minimalinvasiven Operation beobachtete [6]. Die R0-Resektionsrate unterschied sich in keiner der Publikationen. Die R0-Resektionsrate wird in keiner der vier Metaanalysen analysiert.

\section{Onkologisches Outcome}

Bezüglich der RCT liegen bislang keine Überlebensdaten vor [2]. Die verfügbaren Überlebensdaten in den anderen $\mathrm{Pu}-$ blikationen zeigen keine Unterschiede zwischen minimalinvasiver und offener Chirurgie. Zwei von vier Metaanalysen überprüften das 3-Jahres-Überleben und fanden keinen Unterschied zwischen minimalinvasiver und offener Chirurgie [4, 6].

\section{Postoperative Lebensqualität}

Die RCT berichtete über eine bessere früh-postoperative (6 Wochen) Lebensqualität nach minimalinvasiver Chirurgie im Vergleich zur offenen Chirurgie [2]. Daten zu längerfristigen Unterschieden liegen nicht vor. Keine der Metaanalysen analysierte diesen Parameter.

\section{Kosten}

Die Krankenhauskosten wurden in keiner der hier eingeschlossenen Publikationen untersucht. Es wurde allerdings festgestellt, dass zumindest in den USA die Operationskosten der minimalinvasiven Operation diejenigen der offenen Operation bei Weitem überschreiten und diese Mehrkosten auch durch einen kürzeren Krankenhausaufenthalt nicht kompensiert werden [25].

\section{Diskussion und Schlussfolgerungen}

Die minimalinvasive Chirurgie des Ösophagus wurde erstmals 1992 in der Literatur erwähnt [26]. Technisch wurde sie seitdem von vielen Zentren in verschiedenen Aspekten modifiziert und in den letzten Jahren auch zunehmend für Malignome des Ösophagus eingesetzt. Viele Fallberichte aus dieser Zeit belegen, dass die minimalinvasive Ösophagektomie nach einer entsprechenden Lernkurve - mit zumindest vergleichbarer Morbidität und Mortalität wie die offene Ösophagektomie durchgeführt werden kann. Dennoch hat es 20 Jahre gedauert, bis im vergangenen Jahr die erste RCT zum Vergleich der minimalinvasiven mit der offenen Chirurgie bei Ösophaguskarzinomen erschienen ist [2]. Diese RCT war jedoch dringend nötig, da der Evidenzlevel für die minimalinvasive Chirurgie von Ösophaguskarzinomen gering war und methodisch bedingt bei retrospektiven Kohortenstudien immer ein Selektionsbias sowie Dokumentationslücken angenom- 
men werden müssen. Somit kann erst jetzt mit entsprechender Evidenz (wenngleich mit nur einer einzigen RCT) belegt werden, dass auch die minimalinvasive Chirurgie am Ösophagus mit den Vorteilen einhergeht, die von minimalinvasiven Operationen an anderen Organen bekannt und zu erwarten sind: weniger postoperative Schmerzen, kürzerer Krankenhausaufenthalt und höhere früh-postoperative Lebensqualität. Diese Parameter beeinflussen sich gegenseitig und können letztlich alle direkt auf die Reduktion des Zugangswegtraumas bei minimalinvasiven Operationen zurückgeführt werden.

Schwieriger verhält es sich mit der Beobachtung der RCT sowie einiger Kohortenstudien, dass die minimalinvasive Ösophagektomie mit weniger pulmonalen Komplikationen einhergeht. Auch hier kann argumentiert werden, dass die minimalinvasive Chirurgie durch ein geringeres Zugangswegtrauma und weniger postoperative Schmerzen direkt zu einer verbesserten Atemmechanik und Compliance des Patienten beitragen und somit pulmonalen Komplikationen vorbeugt. Mindestens ebenso entscheidend scheint für das Auftreten von postoperativen pulmonalen Komplikationen jedoch auch die intraoperative Beatmungstechnik sowie die Lagerung des Patienten zu sein. So traten zum einen auch bei minimalinvasiver Ösophagektomie mehr pulmonale Komplikationen auf, wenn sie in Linksseitenlagerung mit Ein-Lungen-Beatmung und nicht in Bauchlagerung mit erhaltener Zwei-LungenBeatmung durchgeführt wurde [27, 28]. Zum anderen korrelieren gerade die Ein-Lungen-Beatmung und die Beatmungstechnik direkt mit postoperativen pulmonalen Komplikationen [29-31]. Eine Reduktion von pulmonalen Komplikationen ist also insbesondere durch eine Optimierung der Beatmungstechnik, vor allem bei Vermeidung oder zumindest Verkürzung sowohl der Ein-Lungen-Beatmung als auch der manuellen oder instrumentellen Lungenkompression, möglich. Diese Maßnahmen sind keineswegs nur der minimalinvasiven Chirurgie vorbehalten, sondern sollten auch in der offenen Chirurgie konsequent beachtet werden. In diesem $\mathrm{Zu}$ sammenhang sind auch die langen Operationszeiten von $5 \mathrm{~h}$ und mehr für die offenen Resektionen in vielen Publikationen kritisch anzumerken $[2,7,9,14,15,24]$.

Auf der Grundlage der zur Verfügung stehenden Literatur ist der onkologische Stellenwert der minimalinvasiven Ösophagektomie nicht ausreichend zu beurteilen. Während für die R0Resektionsraten und die Anzahl an entfernten Lymphknoten weitestgehend vergleichbare Ergebnisse für die minimalinvasive und die offene Chirurgie auch in der RCT vorliegen, sind nur sehr wenige Daten für den onkologischen Langzeitverlauf aus retrospektiven Kohortenstudien bzw. keinerlei Daten aus RCTs verfügbar. Gerade bei diesen onkologischen Parametern wird die geringe Qualität der aktuellen Datenlage deutlich. So bestehen zwischen und zum Teil auch innerhalb der Publikationen erhebliche Unterschiede unter anderem in der Stadienverteilung, der Verteilung der Tumortypen (Plattenepithelkarzinom, Adenokarzinom, undifferenzierte Malignome), der Durchführung einer multimodalen Therapie, der Angabe des Überlebens (krankheitsfreies Überleben, Gesamtüberleben) sowie der Qualität und Länge der Nachbeobachtungszeit. Während der Resektionsstatus als robuster Parameter für die chirurgisch-onkologische Qualität angesehen werden kann, gilt dies bei der Anzahl an entfernten Lymphknoten beim Ösophaguskarzinom nur eingeschränkt. Die bloße Anzahl der entfernten Lymphknoten bildet die Qualität einer zumindest abdominellen und mediastinalen sowie potenziell zervikal erforderlichen Lymphadenektomie nur bedingt ab. Erschwert wird ein valider Vergleich der onkologischen Qualität von minimalinvasiver Chirurgie mit der offenen Chirurgie bei Ösophaguskarzinomen zudem durch die Tatsache, dass keine chirurgische Standardtherapie für Ösophaguskarzinome definiert ist. Das in den vorliegenden Publikationen herangezogene Vergleichskollektiv an offen operierten Patienten wurde alles andere als einheitlich operiert - weder bezüglich des Ausmaßes der Resektion und der Lymphadenektomie noch bezüglich der Art und des Orts der Rekonstruktion. Ohne einen definierten und überprüfbaren Standard wird die Evaluation einer neuen Operationstechnik (minimalinvasive Chirurgie) aufgrund der Heterogenität des Kontrollkollektivs (offene Chirurgie) immer zumindest angreifbar bleiben. Die onkologische Qualität zweier nicht standardisierter Verfahren ist somit derzeit kaum vergleichbar. Hier müssen weitere Daten aus RCTs mit homogenen und klar definierten Prozeduren abgewartet werden.

Abschließend dürfen in der Gesamtbeurteilung von minimalinvasiver versus offener Chirurgie auch die Gesamtkosten der Behandlung nicht außer Acht gelassen werden, insbesondere in DRG-basierten Vergütungssystemen. Hier wird die minimalinvasive Chirurgie auch in Zukunft deutlich kostenintensiver bleiben.

\section{Disclosure Statement}

Keine Interessenkonflikte bei beiden Autoren.

\section{Literatur}

1 Sackett DL: Rules of evidence and clinical recommendations on the use of antithrombotic agents. Chest 1989;95(suppl):2-4.
2 Biere SS, van Berge Henegouwen MI, Maas KW, Bonavina L, Rosman C, Garcia JR, Gisbertz SS, Klinkenbijl JH, Hollmann MW, de Lange ES, Bonjer HJ, van der Peet DL, Cuesta MA: Minimally invasive versus open oesophagectomy for patients with oesophageal cancer: a multicentre, open-label, randomised controlled trial. Lancet 2012;379:1887-1892.
3 Biere SS, Cuesta MA, van der Peet DL: Minimally invasive versus open esophagectomy for cancer: a systematic review and meta-analysis. Minerva Chir 2009;64:121-133. 
4 Sgourakis G, Gockel I, Radtke A, Musholt TJ, Timm S, Rink A, Tsiamis A, Karaliotas C, Lang H: Minimally invasive versus open esophagectomy: meta-analysis of outcomes. Dig Dis Sci 2010;55: 3031-3040.

5 Nagpal K, Ahmed K, Vats A, Yakoub D, James D, Ashrafian H, Darzi A, Moorthy K, Athanasiou T: Is minimally invasive surgery beneficial in the management of esophageal cancer? A meta-analysis. Surg Endosc 2010;24:1621-1629.

6 Dantoc M, Cox MR, Eslick GD: Evidence to support the use of minimally invasive esophagectomy for esophageal cancer: a meta-analysis. Arch Surg 2012;147:768-776.

7 Nguyen NT, Follette DM, Wolfe BM, Schneider PD, Roberts P, Goodnight JE Jr: Comparison of minimally invasive esophagectomy with transthoracic and transhiatal esophagectomy. Arch Surg 2000;135:920-925.

8 Osugi H, Takemura M, Higashino M, Takada N, Lee S, Kinoshita H: A comparison of videoassisted thoracoscopic oesophagectomy and radical lymph node dissection for squamous cell cancer of the oesophagus with open operation. Br J Surg 2003;90:108-113.

9 Smithers BM, Gotley DC, Martin I, Thomas JM Comparison of the outcomes between open and minimally invasive esophagectomy. Ann Surg 2007; 245:232-240.

10 Fabian T, Martin JT, McKelvey AA, Federico JA Minimally invasive esophagectomy: a teaching hospital's first year experience. Dis Esophagus 2008; 21:220-225.

-11 Parameswaran R, Veeramootoo D, Krishnadas R, Cooper M, Berrisford R, Wajed S: Comparative experience of open and minimally invasive esophagogastric resection. World J Surg 2009;33:18681875.

12 Zingg U, McQuinn A, DiValentino D, Esterman AJ, Bessell JR, Thompson SK, Jamieson GG, Watson DI: Minimally invasive versus open esophagectomy for patients with esophageal cancer. Ann Thorac Surg 2009;87:911-919.

13 Lazzarino AI, Nagpal K, Bottle A, Faiz O, Moorthy K, Aylin P: Open versus minimally invasive esophagectomy. Trends of utilization and associated outcomes in England. Ann Surg 2010;252:292-298.
14 Schoppmann SF, Prager G, Langer FB, Riegler FM, Kabon B, Fleischmann E, Zacherl J: Open versus minimally invasive esophagectomy: a singlecenter case controlled study. Surg Endosc 2010;24: 3044-3053.

15 Pham TH, Perry KA, Dolan JP, Schipper P, Sukumar M, Sheppard BC, Hunter JG: Comparison of perioperative outcomes after combined thoracoscopic-laparoscopic esophagectomy and open IvorLewis esophagectomy. Am J Surg 2010;199:594-598. 16 Singh RK, Pham TH, Diggs BS, Perkins S, Hunter JG: Minimally invasive esophagectomy provides equivalent oncologic outcomes to open esophagectomy for locally advanced (stage II or III) esophageal carcinoma. Arch Surg 2011;146:711-714.

17 Berger AC, Bloommenthal A, Weksler B, Evans $\mathrm{N}$, Chojnacki KA, Yeo CJ, Rosato EL: Oncologic efficacy is not compromised, and may be improved with minimally invasive esophagectomy. J Am Coll Surg 2011;212:560-568.

18 Gao Y, Wang Y, Chen L, Zhao Y: Comparison of open three-field and minimally-invasive esophagectomy for esophageal cancer. Interact Cardiovasc Thorac Surg 2011;12:366-369.

19 Nafteux P, Moons J, Coosemans W, Decaluwé H, Decker G, De Leyn P, Van Raemdonck D, Lerut T: Minimally invasive esophagectomy: a valuable alternative to open oesophagectomy for the treatment of early oesophageal and gastro-oesophageal junction carcinoma. Eur J Cardio Thorac Surg 2011:40:1455-1465.

20 Ben-David K, Sarosi GA, Cendan JC, Howard D, Rossidis G, Hochwald SN: Decreasing morbidity and mortality in 100 consecutive minimally invasive esophagectomies. Surg Endosc 2012;26:162-167.

21 Kinjo Y, Kurita N, Nakamura F, Okabe H, Tanaka E, Kataoka Y, Itami A, Sakai Y, Fukuhara S: Effectiveness of combined thoracoscopic-laparoscopic esophagectomy: comparison of postoperative complication and midterm oncological outcomes in patients with esophageal cancer. Surg Endosc 2012;26:381-390.

22 Bakhos CT, Fabian T, Oyasiji TO, Gautam S, Gangadharan SP, Kent MS, Martin J, Critchlow JF, DeCamp MM: Impact of the surgical technique on pulmonary morbidity after esophagectomy. Ann Thorac Surg 2012;93:221-227.
23 Mamidanna R, Bottle A, Aylin P, Faiz O, Hanna GB: Short-term outcomes following open versus minimally invasive esophagectomy for cancer in England. Ann Surg 2012;255:197-203.

24 Sundaram A, Geronimo JC, Willer BL, Hoshino M, Torgersen Z, Juhasz A, Lee TH, Mittal SK Survival and quality of life after minimally invasive esophagectomy: a single-surgeon experience. Surg Endosc 2012;26:168-176.

25 Dhamija A, Dhamija A, Hancock J, McCloskey B, Kim AW, Cetterbeck FC, Boffa DJ: Minimally invasive oesophagectomy more expensive than open despite shorter length of stay. Eur J Cardiothorac Surg 2013;DOI: 10.1093/ejcts/ezt482.

26 Cuschieri A, Shimi S, Banting S: Endoscopic oesophagectomy through a right thoracoscopic approach. J R Coll Surg Edinb 1992;37:7-11.

27 Luketich JD, Pennathur A, Awais O, Levy RM, Keeley S, Shende M, Christie NA, Weksler B, Landreneau RJ, Abbas G, Schuchert MJ, Nason KS: Outcomes after minimally invasive esophagectomy: review of over 1000 patients. Ann Surg 2012; 256:95-103.

28 Yatabe T, Kitagawa H, Yamashita K, Akimori T, Hanazaki K, Yokoyama M: Better postoperative oxygenation in thoracoscopic esophagectomy in prone positioning. J Anesth 2010;24:803-806.

29 Michelet P, D'Journo XB, Roch A, Doddoli C, Marin V, Papazian L, Decamps I, Bregeon F, Thomas P, Auffray JP: Protective ventilation influences systemic inflammation after esophagectomy: a randomized controlled study. Anesthesiology 2006;105:911-919.

30 Pelosi P, de Abreu MG: Tidal volumes during general anesthesia: size does matter! Anesthesiology 2012;116:985-986.

31 De Conno E, Steurer MP, Wittlinger M, Zalunardo MP, Weder W, Schneiter D, Schimmer RC, Klaghofer R, Neff TA, Schmid ER, Spahn DR, Z'graggen BR, Urner M, Beck-Schimmer B: Anesthetic-induced improvement of the inflammatory response to one-lung ventilation. Anesthesiology 2009;110:1316-1326. 